\title{
Geraniin Protects against Cerebral Ischemia/Reperfusion Injury by Suppressing Oxidative Stress and Neuronal Apoptosis via Regulation of the Nrf2/HO-1 Pathway
}

\author{
Yuan Yang, Bo He, Xiaochao Zhang, Renhua Yang, Xin Xia, Lu Chen, Rui Li, \\ Zhiqiang Shen $\mathbb{D}$, and Peng Chen $(1 D)$ \\ School of Pharmaceutical Sciences and Yunnan Key Laboratory of Pharmacology for Natural Products, \\ Kunming Medical University, Kunming 650500, China \\ Correspondence should be addressed to Zhiqiang Shen; shzhq21cn@qq.com and Peng Chen; chenpeng@kmmu.edu.cn
}

Received 29 November 2021; Accepted 27 January 2022; Published 18 February 2022

Academic Editor: Vladimir Jakovljevic

Copyright (c) 2022 Yuan Yang et al. This is an open access article distributed under the Creative Commons Attribution License, which permits unrestricted use, distribution, and reproduction in any medium, provided the original work is properly cited.

\begin{abstract}
Geraniin, a polyphenol isolated from Phyllanthus amarus, possesses extensive biological and pharmaceutical activities. In this study, we investigated the protective effect against cerebral ischemia/reperfusion (I/R) injury of geraniin and explored its potential mechanism. Middle cerebral artery occlusion/reperfusion (MCAO/R) was used to simulate cerebral I/R injury in vivo, and oxygen-glucose deprivation/reoxygenation (OGD/R) was applied to establish an in vitro model of cerebral I/R injury. In this study, we performed TTC and HE staining and adopted a neurological score method to evaluate the neuroprotective effect of geraniin in vivo and used the CCK- 8 assay to assess this effect in vitro. Indices of reactive oxidation capacity were measured in vivo and in vitro to verify the antioxidant capacity of geraniin. TUNEL staining and flow cytometry were applied to measure the apoptosis rate, and Western blotting was performed to assess the expression of apoptosis-related proteins. Finally, the expression of $\mathrm{Nrf2}$ and HO-1 was evaluated in vivo and in vitro by Western blotting. Geraniin significantly reduced the infarct volume, decreased neurological deficit scores, alleviated pathological changes in neurons, and increased the cell survival rate. Geraniin increased the activity of superoxide dismutase (SOD) and decreased the activity of lactate dehydrogenase (LDH) and the contents of malondialdehyde (MDA), nitric oxide (NO), and neuronal nitric oxide synthase (nNOS) in vivo and in vitro. In addition, geraniin significantly reduced the apoptosis. Furthermore, geraniin also evidently increased Nrf2 (total and nuclear) and HO-1 protein expression in vivo and in vitro. Collectively, these results imply that geraniin may exert a protective effect against cerebral I/R injury by suppressing oxidative stress and neuronal apoptosis. The mechanism underlying the protective effect of geraniin is associated with activation of the $\mathrm{Nrf} 2 / \mathrm{HO}-1$ pathway. Our results indicate that geraniin may be a potential drug candidate for the treatment of ischemic stroke.
\end{abstract}

\section{Introduction}

Stroke, a common cerebrovascular disease in the clinic, can be divided into ischemic and hemorrhagic stroke and remains the predominant cause of death and disability in both developed and developing countries [1]. Ischemic stroke, which is caused by sudden interruption of blood flow to the brain, resulting in brain cell death and neurological impairment, is the most prevalent type of stroke and accounts for approximately $75 \%$ of all stroke cases [2]. Currently, early restoration of blood supply after ischemic stroke is considered the main treatment strategy for improving clinical outcomes. However, the reperfusion processes may further exacerbate the initial ischemic injury after ischemic attack, and this process is termed cerebral ischemia/reperfusion (I/R) injury [3]. Although much progress has been made in the study of the mechanism underlying cerebral I/ $\mathrm{R}$ injury in recent years, the pathogenesis of the disease is still not fully elucidated, and there is a lack of effective treatments [4].

Oxidative stress is one of the most important processes in cerebral $\mathrm{I} / \mathrm{R}$ injury and is also a major risk factor for cell 
apoptosis [5]. Studies have shown that oxidative stress is elevated in rat brain tissue after middle cerebral artery occlusion/reperfusion- (MCAO/R-) induced injury, as the activity of superoxide dismutase (SOD) is decreased, the activity of lactate dehydrogenase (LDH) is increased, and the contents of oxidative stress markers such as malondialdehyde (MDA), nitric oxide (NO), and neuronal nitric oxide synthase (nNOS) are increased [6]. Moreover, excessive accumulation of free radicals can lead to damage to macromolecules such as proteins and DNA and lipid peroxidation and accelerate neuronal apoptosis during the development of cerebral I/R injury [7]. Oxidative stress is closely associated with cell apoptosis, which can trigger the cell death pathway involving Bcl-2 family proteins and subsequently contribute to activation of the caspase cascade and proteolysis [8]. Sun and Cui found that cerebral I/R injury significantly reduces the activity of the antioxidant enzyme SOD, increases the content of prooxidant factors such as MDA, and exerts proapoptotic effects by upregulating Bax and caspase-3 expression and downregulating Bcl-2 expression [9]. These studies indicate that oxidative stress and cell apoptosis play important roles in the pathogenesis of cerebral I/ $\mathrm{R}$ injury. Therefore, antioxidant and antiapoptotic agents have been the focus of studies on the use of neuroprotective drugs for the prevention and treatment of cerebral stroke.

Previous studies have revealed that decreased expression of nuclear factor E2-related factor 2 (Nrf2) and heme oxygenase-1 (HO-1) contributes to aggravation of brain damage by increasing the infarct volume and decreasing neurological function, during cerebral ischemia [10]. Many previous studies have shown that natural products or Chinese herbal medicines exert neuroprotective effects against cerebral ischemia by activating the Nrf2/HO- 1 pathway. Fu et al. found that pelargonidin can effectively reduce the infarct volume and improve neurological function in rats subjected to middle cerebral artery occlusion (MCAO), thereby enhancing memory and learning, and that the potential mechanism is associated with activation of the Nrf2/HO-1 signaling pathway [11]. Thus, the Nrf2/HO-1 signaling pathway is a key protective pathway against oxidative stress and apoptosis.

Geraniin is a polyphenol isolated from the medicinal plant Phyllanthus amarus. Geraniin has antioxidative, anti-inflammatory, antithrombotic, and other biological activities. Studies have reported that geraniin protects bone marrow-derived mesenchymal stem cells from $\mathrm{H}_{2} \mathrm{O}_{2}$-induced oxidative stress injury via the PI3K/Akt pathway [12]. Another study revealed that geraniin mitigates lipopolysaccharide- (LPS-) elicited neural/synaptic neurodegeneration, amyloidogenesis, neuroinflammation, and cognitive impairment and suggested geraniin as a therapeutic option for neuroinflammationassociated neurological disorders such as Alzheimer's disease [13]. In addition, we performed some studies on geraniin and found that geraniin can inhibit platelet aggregation, suppress platelet-neutrophil interactions, and exert an antithrombotic effect [14]. Moreover, our previous studies indicated that geraniin has antiosteoporotic effects, promoting the osteogenic differentiation of osteoporotic large bone marrow mesenchymal stem cells in vitro and increasing the expression of Wnt3a and $\beta$-catenin [15]. Although there have been many reports on the pharmacological effects of geraniin, research on the neuroprotective effects of geraniin and its molecular mechanism is limited.

In this study, we adopted a rat model of $\mathrm{MCAO} / \mathrm{R}$ and a PC12 cell model of oxygen-glucose deprivation/reperfusion(OGD/R-) induced oxidative stress injury to investigate the protective effect of geraniin and its potential molecular mechanism of action. This study provides a theoretical and experimental basis for geraniin as a new therapeutic drug for stroke and oxidative stress-related diseases.

\section{Materials and Methods}

2.1. Chemical Reagents. Geraniin (purity > 99\%) was kindly provided by Professor Jikai Liu (Kunming Institute of Botany, Chinese Academy of Science, Kunming, China). Nimodipine injection was obtained from Bayer Pharmaceutical Co., Ltd. (Guangzhou, China). 2,3,5-Triphenyltetrazolium chloride (TTC) was purchased from Solarbio Science \& Technology Co., Ltd. (Beijing, China). SOD and MDA, $\mathrm{LDH}, \mathrm{NO}$, and nNOS kits were obtained from Nanjing Jiancheng Bioengineering Institute (Nanjing, China). A TdT In Situ Apoptosis Detection Kit was purchased from R\&D Systems, Inc. (Minneapolis, USA). An Annexin V-FITC Apoptosis Detection Kit was obtained from KeyGEN Biotech Co., Ltd. (Nanjing, China). Dulbecco's modified Eagle's medium (DMEM), fetal bovine serum (FBS), and penicillin-streptomycin were obtained from Gibco (Grand Island, NY, USA). All other chemicals and solvents used were of either analytical grade or pharmaceutical grade.

2.2. Animal Experiments and Ethics Statement. Male specific pathogen-free- (SPF-) grade Sprague-Dawley (SD) rats weighing 280-320 g were provided by the Laboratory Animal Center of Kunming Medical University (license number: SCXK (Yunnan) k2020-0006). The animals were housed on a $12 / 12 \mathrm{~h} \mathrm{light/dark}$ cycle at room temperature $\left(24 \pm 1^{\circ} \mathrm{C}\right)$. The rats were provided free access to rodent diet and tap water and subjected to adaptive feeding for one week. All procedures were conducted in strict accordance with the Chinese Legislation on the Use and Care of Laboratory Animals and approved by the Medical Ethical Committee of Kunming Medical University. The MCAO/R model was established as previously described by our group [16]. Briefly, after anesthetization with $2 \%$ isoflurane, the rats were fixed to an operating plate in the supine position, and the hair above the cervical spine was carefully removed with an electric razor. Then, the right common carotid artery (CCA) and external carotid artery (ECA) were isolated and ligated. A monofilament nylon suture with a rounded tip (diameter: $0.47 \mathrm{~mm}$ ) was gently inserted into the internal carotid artery (ICA) through the ECA to occlude the origin of the middle cerebral artery. During the operation, the rats were placed on a heating pad, and the thread was removed after $2 \mathrm{~h}$ of ischemia to allow reperfusion for $72 \mathrm{~h}$.

2.3. Experimental Groups. All rats were randomly assigned to the following six groups $(n=8$ each): (1) the shamoperated (sham) group, (2) the $\mathrm{MCAO} / \mathrm{R}$ group, (3) the 
$5 \mathrm{mg} / \mathrm{kg}$ geraniin-treated group, (4) the $10 \mathrm{mg} / \mathrm{kg}$ geraniintreated group, (5) the $20 \mathrm{mg} / \mathrm{kg}$ geraniin-treated group, and (6) the $1 \mathrm{mg} / \mathrm{kg}$ nimodipine-treated group. Geraniin was dissolved in dimethyl sulfoxide (DMSO) and diluted to different concentrations with normal saline. The sham-operated rats underwent the same protocol but without MCA ligation. Two hours after the cerebral ischemia, geraniin $(5,10$, and $20 \mathrm{mg} / \mathrm{kg} \cdot \mathrm{d})$ and nimodipine $(1 \mathrm{mg} / \mathrm{kg} \cdot \mathrm{d})$ were intraperitoneally injected into the rats, and the agents were administered continuously for three days.

2.4. Neurological Deficit Evaluation. After reperfusion for $72 \mathrm{~h}$, neurological deficits were assessed according to the following scoring criteria [17]: 0, no neurological deficits; 1 , unable to extend the contralateral forelimb; 2, circling to the paretic side; 3 , falling to the contralateral side; and 4, unable to engage in spontaneous activity.

2.5. Infarct Volume Measurement. To determine the effect of geraniin on brain infarction, intact brains were quickly removed after neurological deficit evaluation. The brains were cut into five $2 \mathrm{~mm}$ thick coronal sections and incubated for $30 \mathrm{~min}$ in $2 \%$ TTC solution at $37^{\circ} \mathrm{C}$. All images were collected and analyzed using ImageJ (NIH, USA) [18].

2.6. Hematoxylin and Eosin (H\&E) Staining. The rats were anesthetized $72 \mathrm{~h}$ after $\mathrm{MCAO} / \mathrm{R}$ and perfused with $4 \%$ paraformaldehyde. Next, the brains were rapidly removed and fixed with $4 \%$ paraformaldehyde overnight. The brains were then embedded in paraffin and cut into $6 \mu \mathrm{m}$ thick sections. Subsequently, the sections were deparaffinized in xylene and rehydrated through graded alcohol solutions. Then, the sections were stained with HE. Morphological changes in cerebral cortex and hippocampal tissues were observed under a light microscope (Olympus, Tokyo, Japan).

\subsection{Terminal Deoxynucleotidyl Transferase- (TdT-)} Mediated d-UTP Nick End Labeling (TUNEL) Staining. TUNEL staining was performed to determine the cell apoptosis rate as previously described [19]. Brains were sliced into $4 \mu \mathrm{m}$ thick frozen sections. The number of TUNELpositive cells in the cerebral cortex and hippocampus was counted in four randomly selected rats from each group. The number of TUNEL-positive cells was determined by an observer blinded to the study design. Images were collected using an automatic fluorescence microscope and analyzed using ImageJ software (NIH, USA).

\subsection{Oxidative Stress Measurement}

2.8.1. SOD Activity. SOD activity in the serum, brain tissues, and PC12 cells was measured according to the instructions of the kit. Substrate application solution and enzyme working solution were prepared, and the samples to be tested were added to these solutions and incubated at $37^{\circ} \mathrm{C}$ for $20 \mathrm{~min}$. An enzyme immunoassay reader was used to measure the absorbance at $450 \mathrm{~nm}$. SOD activity (U/L) was calculated as the SOD inhibition rate $/ 50 \% \times 2 /$ the concentration of the tested sample.

2.8.2. LDH Activity. Working solution was prepared according to the instructions of the kit, and the sample to be tested was added and incubated at room temperature for $5 \mathrm{~min}$. An enzyme immunoassay reader was used to measure the absorbance at $450 \mathrm{~nm}$. LDH activity (U/L) was calculated as ( test OD - control OD $) /($ standard OD - negative control OD) $\times$ the concentration of the standard $(0.2 \mathrm{nmol} / \mathrm{mL}) \times 1000$.

2.8.3. MDA Content. Working solution was prepared according to the instructions of the kit, and the samples to be tested were added and incubated in water at $95^{\circ} \mathrm{C}$ for $1 \mathrm{~h}$. After removal, the samples were cooled with running water and centrifuged at $4000 \mathrm{r} / \mathrm{min}$ for $10 \mathrm{~min}$. The supernatant was collected for analysis. An enzyme immunoassay reader was used to measure the absorbance at $532 \mathrm{~nm}$. MDA content was calculated as (test OD - controlOD)/( standard OD - negative control OD) $\times$ the concentration of the standard $(10 \mathrm{nmol} / \mathrm{mL})$.

2.8.4. NO Content. Working solution was prepared according to the instructions of the kit, and the samples to be tested were added and incubated at room temperature for $15 \mathrm{~min}$. An enzyme immunoassay reader was used to measure the absorbance at $550 \mathrm{~nm}$. NO content $(\mu \mathrm{mol} / \mathrm{L})$ was calculated as (test OD - control OD)/(standard OD - negative control OD) $\times 20 \mu \mathrm{mol} / \mathrm{L} \times 2 /$ the concentration of the tested sample.

2.8.5. nNOS Content. Working solution was prepared according to the instructions of the kit, and the samples to be tested were added and incubated at $37^{\circ} \mathrm{C}$ for $30 \mathrm{~min}$. An enzyme immunoassay reader was used to measure the absorbance at $450 \mathrm{~nm}$. According to the kit instructions, ELISA calc software was used for calculation, and a logistic curve was selected for fitting the model.

2.9. Cell Culture and Treatment. PC12 cells were purchased from the Kunming Institute of Zoology, Chinese Academy of Science. They were cultured in DMEM1640 supplemented with $10 \%$ FBS and $1 \%$ penicillin-streptomycin and incubated at $37^{\circ} \mathrm{C}$ in $5 \% \mathrm{CO}_{2}$ and $95 \% \mathrm{O}_{2}$. To mimic the cerebral I/R injury, an in vitro cell model of OGD/R was established as previously described [20]. First, PC12 cells incubated with glucose-free medium were placed in an incubator at $37^{\circ} \mathrm{C}, 1 \% \mathrm{O}_{2}, 95 \% \mathrm{~N}_{2}$, and $5 \% \mathrm{CO}_{2}$ (SHel Lab, China). Two hours after oxygen-glucose deprivation (OGD), the PC12 cells were placed in a normal incubator, and the glucose-free medium was removed. The medium of the cells in the OGD/R model group was replaced with fresh complete medium, while that of the drug administration group was replaced with medium containing geraniin at different concentrations $(0.1 \mu \mathrm{mol} / \mathrm{L}, 1 \mu \mathrm{mol} / \mathrm{L}$, and $10 \mu \mathrm{mol} / \mathrm{L})$ or nimodipine $(10 \mu \mathrm{mol} / \mathrm{L})$. After $24 \mathrm{~h}$ of incubation, the cells were collected for subsequent experiments.

2.10. Flow Cytometric Analysis. The cell apoptosis rate was measured after OGD/R by flow cytometry according to the manufacturer's protocol [21]. Briefly, the cells were washed twice with ice-cold PBS and stained with FITC-conjugated Annexin $\mathrm{V}$ and propidium iodide (PI) at room temperature for $15 \mathrm{~min}$ in the dark using an Annexin V-FITC Apoptosis Detection Kit. The stained cells were analyzed using flow cytometry as soon as possible (within $1 \mathrm{~h}$ ). Annexin V- 
FITC+/PI- and Annexin V-FITC+/PI+ cells were considered early apoptotic and late apoptotic cells, respectively. The apoptotic rate was calculated as the percentage of early/primary apoptotic cells (Annexin $\mathrm{V}+/ \mathrm{PI}$ ) and late/secondary apoptotic cells (Annexin V+/PI+) [22].

2.11. Western Blotting. Proteins were extracted from brain tissues of the ischemic side and PC12 cells, separated on $10 \%-15 \%$ SDS-PAGE gels, and transferred to membranes. Then, the membranes were incubated with primary antibodies against $\mathrm{Bax}(1: 1000$, ABclonal, China), Bcl-2 (1:1000, ABclonal, China), caspase-3 (1:1000, ABclonal, China), cleaved caspase-3 (1:1000, ABclonal, China), Nrf2 (1:1000, Cell Signaling Technology, USA), HO-1 (1:1000, Proteintech, USA), Lamin B (1:1000, Abcam, USA), GAPDH $(1: 1000$, Proteintech, USA), and $\beta$-actin $(1: 1000$, Proteintech, USA). After three washes with TBST, the membranes were incubated with corresponding secondary antibodies (1:6500, Abcam, USA) and scanned using a BIORAD imaging system (BIO-RAD Gel Doc XR, USA). The band intensity was normalized to the intensity of the GAPDH or Lamin B band and analyzed using Image Lab v5.2 software.

2.12. Statistical Analysis. The data are expressed as the means \pm SEM. One-way analysis of variance (ANOVA) was used to analyze the significance of differences between groups. $P$ values below 0.05 were considered significant.

\section{Results}

3.1. Geraniin Exerts a Protective Effect against the I/R Injury In Vivo. As shown in Figures 1(b)-1(d), the infarct volume and neurological scores were increased in the MCAO/R group compared to the sham group $(P<0.01)$. In contrast, geraniin $(10$ and $20 \mathrm{mg} / \mathrm{kg})$ and nimodipine $(1 \mathrm{mg} / \mathrm{kg})$ treatment significantly decreased the infarct volume and neurological scores $(P<0.01)$. We also conducted HE staining to observe neuronal morphology in the cerebral cortex and hippocampus. As revealed in Figure 1(e), most neurons in the sham group appeared round, had large nuclei, and were arranged in a regular pattern. In the MCAO/R group, the injured neurons were disarranged and shrunken and had dark nuclei. However, the number of surviving neurons was significantly increased in the geraniin- $(20 \mathrm{mg} / \mathrm{kg})$ and nimodipine- $(1 \mathrm{mg} / \mathrm{kg})$ treated groups compared with the $\mathrm{MCAO} / \mathrm{R}$ group. These results indicated that geraniin protects against $I / R$ injury in rats in vivo.

3.2. Geraniin Suppresses MCAO/R-Induced Oxidative Stress In Vivo. Next, we evaluated the effect of geraniin against oxidative stress in a rat model of $M C A O / R$. As shown in Figure 2, the activity of SOD in the sera and brain tissues in the MCAO/R group was lower than that in the sham group $(P<0.01)$. Compared with that in the $\mathrm{MCAO} / \mathrm{R}$ group, the level of SOD in the geraniin- $(20 \mathrm{mg} / \mathrm{kg})$ and nimodipine- $(1 \mathrm{mg} / \mathrm{kg})$ treated groups was significantly higher $(P<0.05$ or $P<0.01)$. The levels of LDH, MDA, $\mathrm{NO}$, and nNOS in the MCAO/R group were significantly increased compared with those in the sham group
$(P<0.01)$. Geraniin significantly decreased the levels of these molecules in a dose-dependent manner $(P<0.05$ and $P<0.01)$. These results suggest that geraniin treatment can obviously suppress oxidative stress induced by $\mathrm{MCAO} / \mathrm{R}$.

3.3. Geraniin Attenuates MCAO/R-Induced Neuronal Apoptosis In Vivo. To investigate the protective effects of geraniin against $\mathrm{MCAO} / \mathrm{R}$-induced neuronal apoptosis, TUNEL staining and Western blotting were performed. As shown in Figure 3, few TUNEL-positive cells were present in cerebral cortex and hippocampal sections from the sham group. The number of TUNEL-positive cells was more significantly increased in the MCAO/R group than in the sham group $(P<0.01)$. Treatment with geraniin $(20 \mathrm{mg} / \mathrm{kg})$ and nimodipine $(1 \mathrm{mg} / \mathrm{kg})$ significantly decreased the number of TUNEL-positive cells $(P<0.05$ or $P<0.01)$. In addition, compared with that in the sham group, the expression of Bax and cleaved caspase- 3 in the MCAO/R group was significantly enhanced, whereas the expression of Bcl-2 was significantly reduced, resulting in a low Bcl-2/Bax expression ratio $(P<0.05$ or $P<0.01)$. Geraniin decreased the expression of Bax and cleaved caspase- 3 but significantly increased the expression of $\mathrm{Bcl}-2$ in a dose-dependent manner, resulting in a high $\mathrm{Bcl}-2 / \mathrm{Bax}$ expression ratio $(P<0.05$ or $P<0.01)$. These results suggest that geraniin treatment can attenuate $\mathrm{MCAO} / \mathrm{R}$-induced neuronal apoptosis in vivo.

3.4. Geraniin Protects PC12 Cells from OGD/R-Induced Cytotoxicity and Oxidative Stress. To verify the safe concentration range of geraniin in PC12 cells, we treated PC12 cells with different concentrations of geraniin $(0.01,0.1,1,10$, and $100 \mu \mathrm{mol} / \mathrm{L}$ ) for $24 \mathrm{~h}$. As presented in Figure 4(a), the cell survival rate of the geraniin- $(100 \mu \mathrm{mol} / \mathrm{L})$ treated group was significantly decreased compared with that of the control group $(P<0.01)$. Thus, we used a concentration of 0.1 $-10 \mu \mathrm{mol} / \mathrm{L}$ for subsequent studies of the effect of geraniin. $\mathrm{OGD} / \mathrm{R}$ significantly decreased the cell survival rate $(P<0.01)$, indicating cell death. Compared with that of OGD/R-treated cells, the survival rate of the OGD/R-treated PC12 cells administered with geraniin ( 1 and $10 \mu \mathrm{mol} / \mathrm{L})$ was significantly increased in a concentration-dependent manner $(P<0.01)$. Additionally, the level of SOD was significantly lower in the OGD/R group than in the control group $(P<0.01)$. Compared with that in the OGD/R group, the level of SOD in the geraniin- $(10 \mu \mathrm{mol} / \mathrm{L})$ and nimodipine$(10 \mu \mathrm{mol} / \mathrm{L})$ treated groups was significantly higher $(P<0.01)$. As shown in Figures $4(\mathrm{~d})-4(\mathrm{~g})$, the levels of $\mathrm{LDH}, \mathrm{MDA}, \mathrm{NO}$, and nNOS in the OGD/R group were significantly increased compared with those in the control group $(P<0.01)$. In contrast, geraniin reduced the levels of $\mathrm{LDH}, \mathrm{MDA}, \mathrm{NO}$, and nNOS in a concentration-dependent manner $(P<0.05$ or $P<0.01)$. These results indicate that geraniin can protect PC12 cells from OGD/R-induced cytotoxicity and oxidative stress.

3.5. Geraniin Protects PC12 Cells from OGD/R-Induced Cell Apoptosis. We also assessed the effect of geraniin on OGD/ R-induced apoptosis of PC12 cells by flow cytometry and Western blot analysis. As shown in Figures 5(a) and 5(b), 


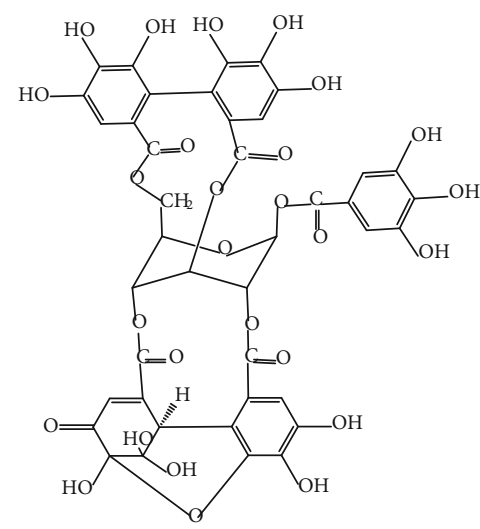

Geraniin

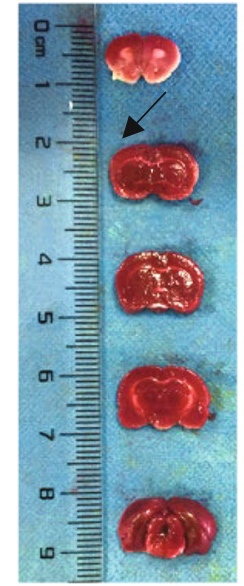

sov
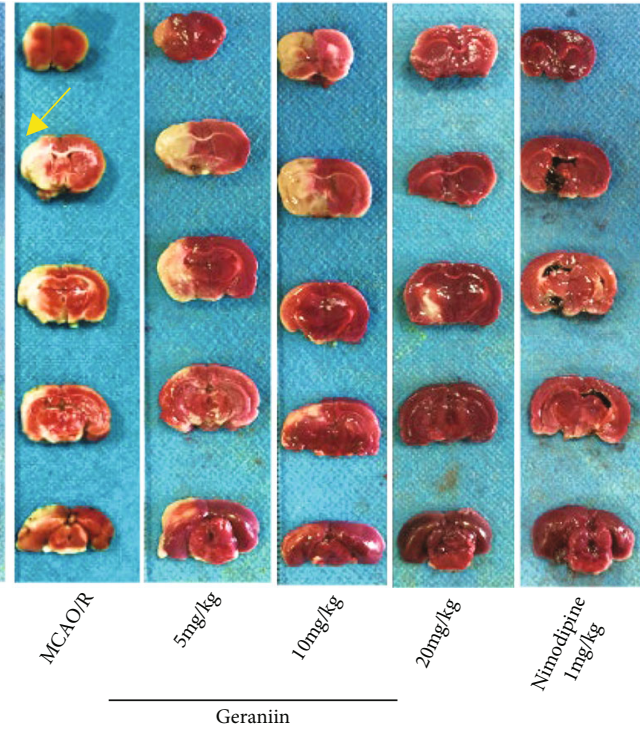

(b)

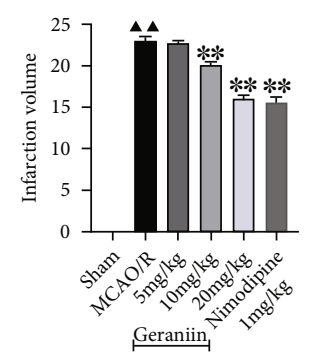

(c)

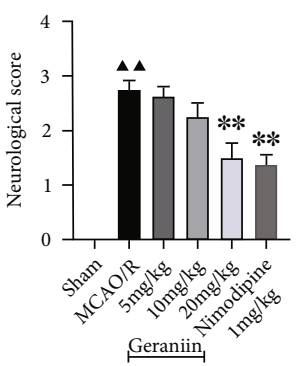

(d)
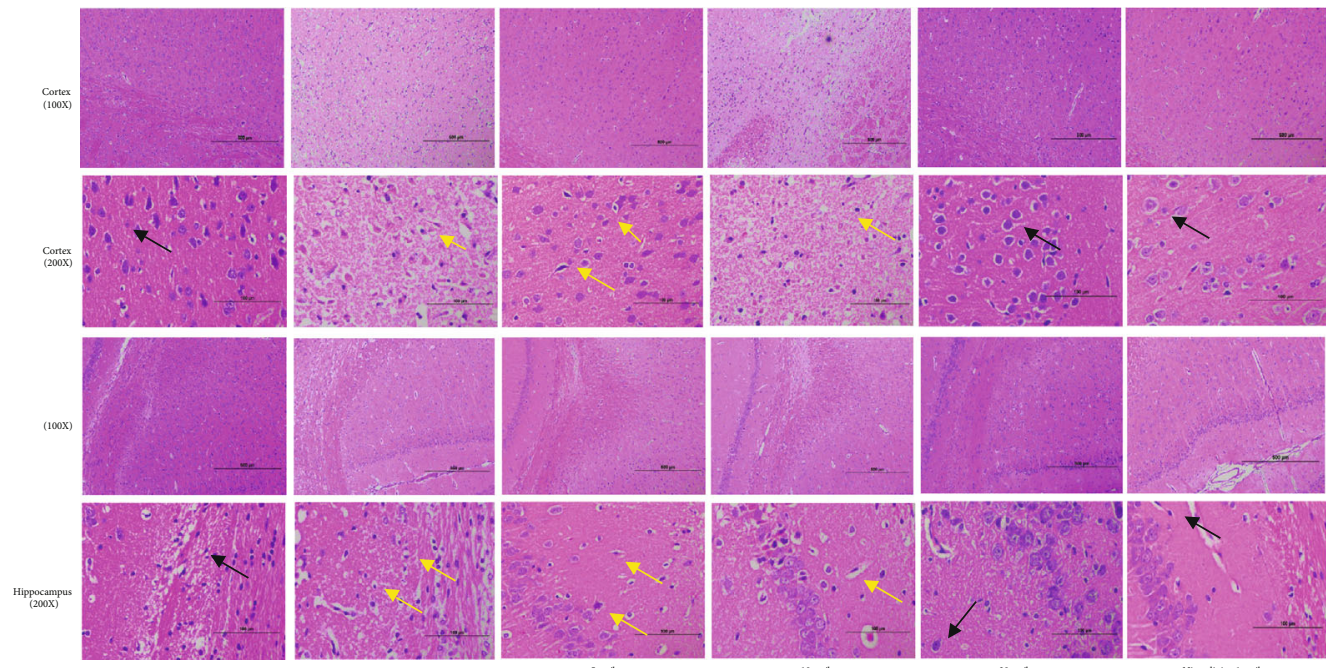

Geraniin

(e)

FIGURE 1: The effect of geraniin on the infarct volume, neurological scores, and neuronal morphology following $72 \mathrm{~h}$ of reperfusion after $2 \mathrm{~h}$ of MCAO. (a) The chemical structure of geraniin. (b) Representative brain sections ( $2 \mathrm{~mm}$ thick) stained with TTC after $72 \mathrm{~h}$ of MCAO and treatment with 5,10 , and $20 \mathrm{mg} / \mathrm{kg}$ geraniin and $1 \mathrm{mg} / \mathrm{kg}$ nimodipine. Normal brain tissue is red and is indicated by the black arrow, while infarcted tissue is pale gray and is indicated by the yellow arrow. (c) Quantitative analysis of the infarct size in each group. (d) Quantitative analysis of neurological scores in each group at $72 \mathrm{~h}$ after reperfusion. (e) Pathological changes in the cerebral cortex and hippocampus were detected by HE staining $(100 \mathrm{x}$, scale bar $=500 \mu \mathrm{m} ; 200 \mathrm{x}$, scale bar $=100 \mu \mathrm{m})$. The black arrows indicate intact nerve cells, and the white arrows indicate damaged nerve cells. The data are expressed as the means \pm SEM $(n=8) .{ }^{\boldsymbol{\Delta}} P<0.01$ vs. the sham group; ${ }^{*} P<0.05,{ }^{* *} P$ $<0.01$ vs. the $\mathrm{MCAO} / \mathrm{R}$ group. 
Serum

(a)

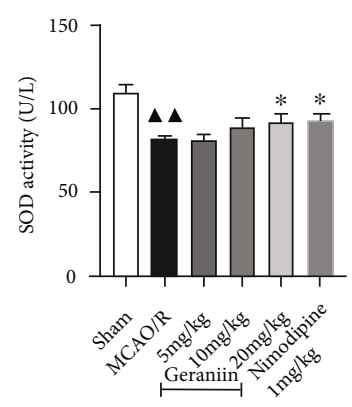

(b)

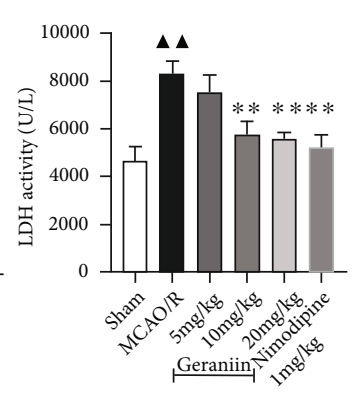

(c)

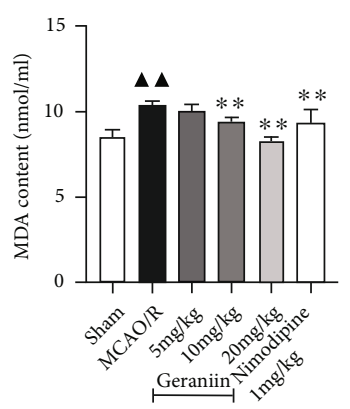

(d)

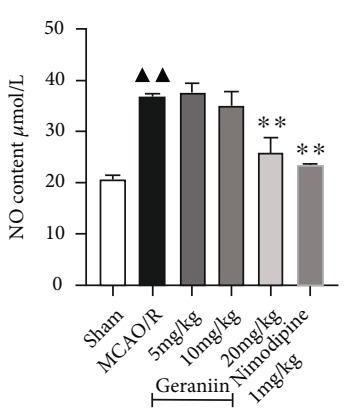

(e)

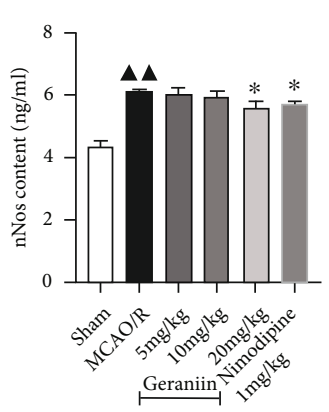

Brain tissue

(f)

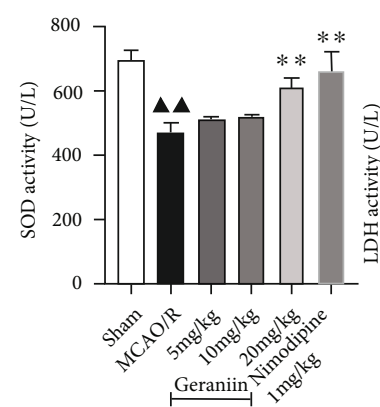

$(\mathrm{g})$

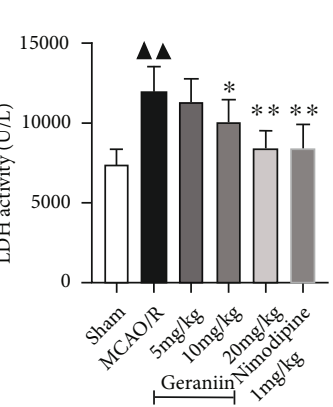

(h)

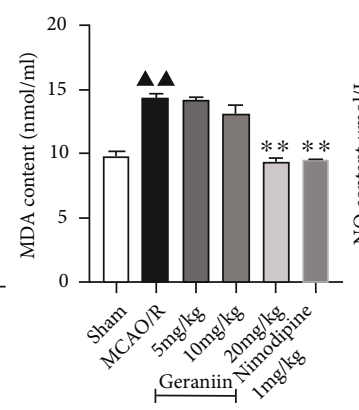

(i)

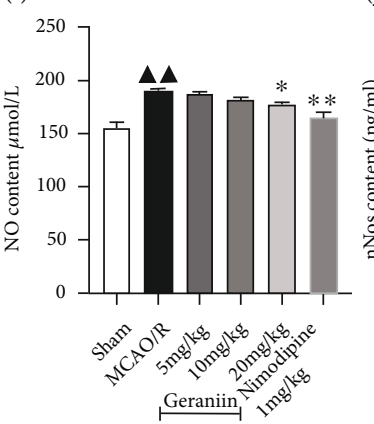

(j)

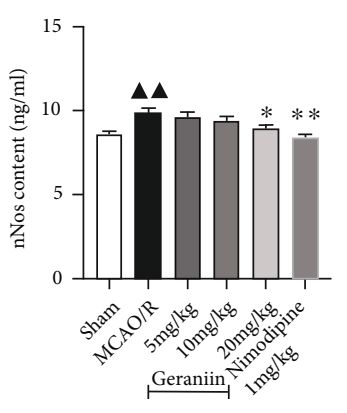

FIGURE 2: Effect of geraniin on oxidative stress $72 \mathrm{~h}$ after MCAO in rats. (a-e) Effects of geraniin on serum SOD and LDH activity and MDA, NO, and nNOS contents. ( $\mathrm{f}-\mathrm{j})$ Effects of geraniin on SOD and LDH activity and MDA, NO, and nNOS contents in brain homogenates from rats. The data are expressed as the means \pm SEM $(n=8)$. ${ }^{\boldsymbol{\Delta}} P<0.01$ vs. the sham group; ${ }^{*} P<0.05,{ }^{* *} P<0.01$ vs. the $\mathrm{MCAO/R}$ group.

the apoptosis rate of PC12 cells increased from $1.2 \%$ to $29.1 \%$ after exposure to OGD/R $(P<0.01)$, whereas treatment with geraniin $(0.1,1$, and $10 \mu \mathrm{mol} / \mathrm{L})$ significantly decreased the cell apoptosis rate after OGD/R in a concentration-dependent manner $(P<0.05$ or $P<0.01)$. Moreover, as presented in Figures 5(c) $-5(\mathrm{~g})$, the expression of apoptosis-related proteins, such as Bax and cleaved caspase-3, was enhanced and Bcl-2 expression was decreased in PC12 cells exposed to OGD/R $(P<0.01)$. Treatment with geraniin $(10 \mu \mathrm{mol} / \mathrm{L})$ and nimodipine $(10 \mu \mathrm{mol} / \mathrm{L})$ markedly reduced the protein expression of Bax and cleaved caspase- 3 and elevated $\mathrm{Bcl}-2$ expression, resulting in a significant increase in the $\mathrm{Bcl}-2 / \mathrm{Bax}$ ratio. These results demonstrate that geraniin can protect PC12 cells from OGD/R-induced cell apoptosis.

3.6. Geraniin Regulates the Nrf2/HO-1 Signaling Pathway In Vivo and In Vitro. To evaluate whether the neuroprotective effect of geraniin is related to the $\mathrm{Nrf} 2 / \mathrm{HO}-1$ signaling pathway, the protein expression of total Nrf2, cytoplasmic Nrf2, nuclear Nrf2, and HO-1 was evaluated by Western blotting. As shown in Figures 6(a)-6(e), compared with that in the sham group, total Nrf2 protein expression in the MCAO/R group was significantly decreased, while the expression of nuclear Nrf2 was increased $(P<0.01)$. However, there was no significant difference in the expression of cytoplasmic
Nrf2 and HO-1 between these two groups. Treatment with geraniin (10 and $20 \mathrm{mg} / \mathrm{kg}$ ) and nimodipine ( $1 \mathrm{mg} / \mathrm{kg})$ markedly decreased the expression of Nrf2 in the cytoplasm in a concentration-dependent manner and increased total Nrf2, nuclear Nrf2, and HO-1 protein expression $(P<0.05$ or $P$ $<0.01)$. Consistent with the in vivo data, the expression of total Nrf2, cytoplasmic Nrf2, nuclear Nrf2, and HO-1 showed similar changes in the geraniin-treated group in vitro. These results indicated that geraniin can facilitate the activation of the $\mathrm{Nrf} 2 / \mathrm{HO}-1$ signaling pathway in vivo and in vitro.

\section{Discussion}

In this study, we confirmed for the first time that geraniin exerts a neuroprotective effect against cerebral I/R injury. Our study demonstrated that treatment with geraniin ameliorated cerebral I/R injury by decreasing the infarct volume and improving neurological scores. Moreover, geraniin administration attenuated oxidative stress and neuronal apoptosis in vivo and in vitro. Furthermore, the neuroprotective effect of geraniin was associated with activation of the Nrf2/HO-1 pathway.

The protective effect of geraniin, as a natural polyphenol, has attracted the attention of a few researchers. Youn and Jun found that geraniin alleviates oxidative damage and 


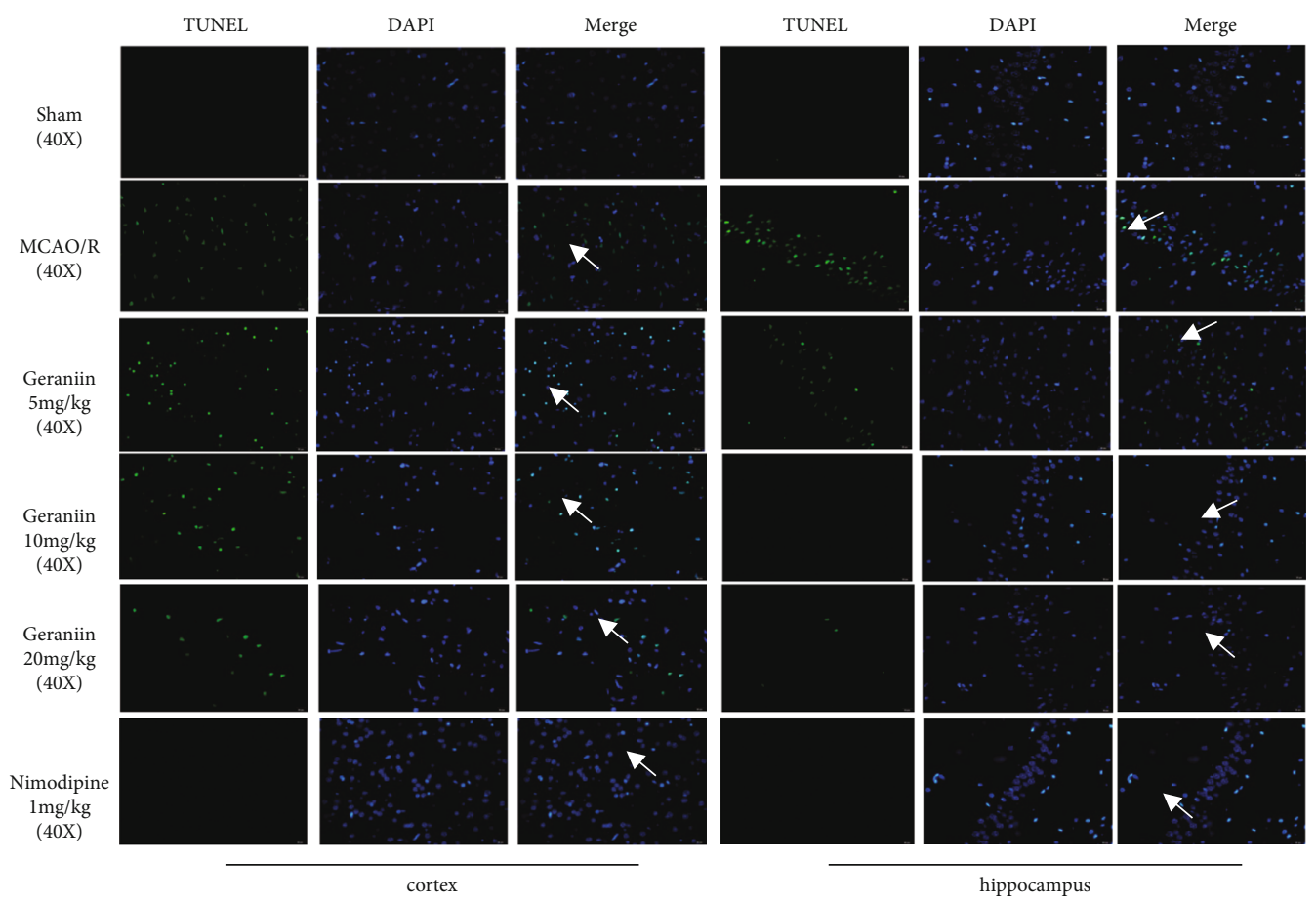

(a)

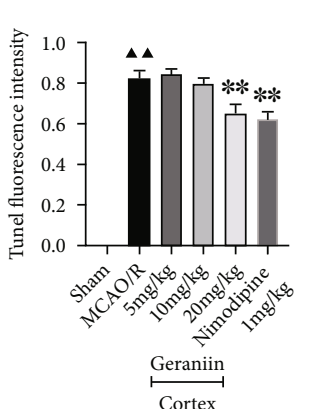

(b)

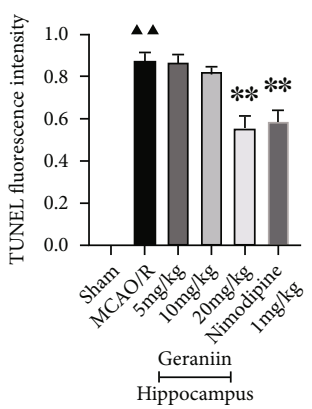

(c)

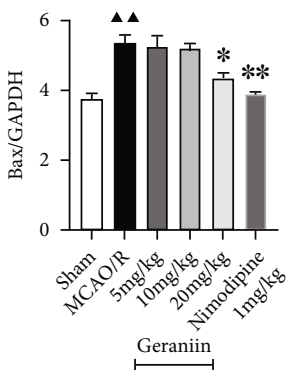

(e)

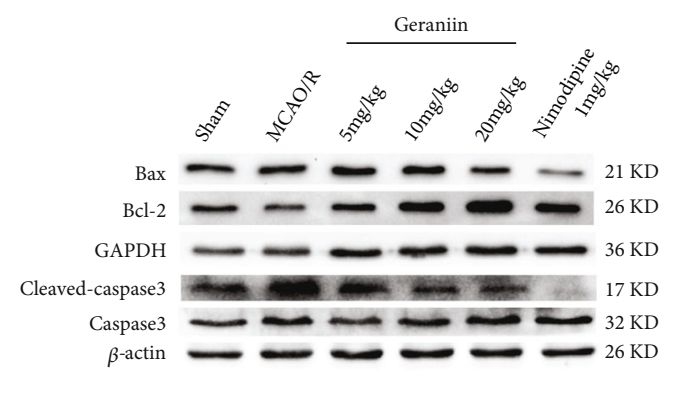

(d)

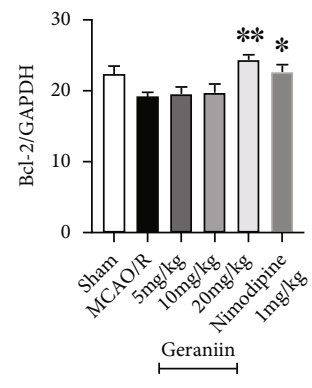

(f)

Figure 3: Continued. 


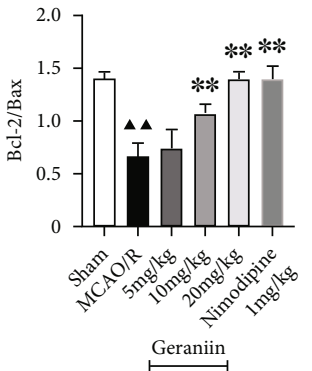

(g)

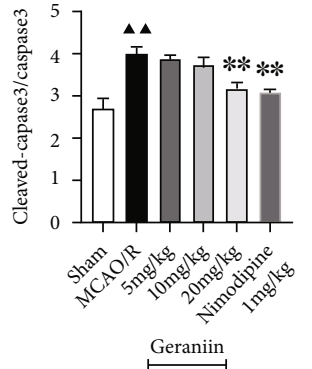

(h)

Figure 3: The effect of geraniin on neuronal apoptosis $72 \mathrm{~h}$ after MCAO in rats. (a-c) Effect of geraniin on MCAO/R-induced neuronal apoptosis in cerebral cortex and hippocampal sections, as determined by TUNEL staining (40x, scale bar $=20 \mu \mathrm{m})(n=4)$. The white arrows indicate TUNEL-positive cells. (d-h) The expression levels of Bax, Bcl-2, cleaved caspase-3, and caspase-3 were measured by Western blot analysis $(n=6)$. The data are expressed as the means \pm SEM. ${ }^{\wedge \boldsymbol{\Delta}} P<0.01$ vs. the sham group; ${ }^{*} P<0.05$, ${ }^{* *} P<0.01$ vs. the $\mathrm{MCAO} / \mathrm{R}$ group.

neuroinflammation in $\mathrm{A} \beta_{25-35}$-treated PC12 cells [23]. Wang et al. reported that geraniin mitigates LPS-elicited neural/ synaptic neurodegeneration, amyloidogenesis, neuroinflammation, and cognitive impairment [24]. However, the protective effect and molecular mechanism of geraniin in ischemic stroke and cerebral I/R injury have not been reported. Our results showed that treatment with geraniin significantly decreased the infarct volume, improved neurological scores, ameliorated pathological changes in neurons in the cortex and hippocampus after $\mathrm{MCAO} / \mathrm{R}$, and increased the survival rate of OGD/R-treated PC12 cells. These results suggest that geraniin exerts a protective effect against cerebral I/R injury.

After cerebral I/R injury, oxygen free radicals are crucial protagonists of oxidative stress and cause neuronal injury and death [25]. Multiple antioxidants have been shown to ameliorate cerebral I/R injury, strongly indicating that suppression of oxidative stress is an attractive potential therapeutic target for counteracting cerebral I/R injury [26]. During cerebral I/R injury, oxygen free radicals are produced, SOD activity is decreased, and LDH activity and MDA content are increased, leading to lipid peroxidation, which further impairs nerve cell function and results in brain damage. At this time, nNOS, the level of which is increased, catalyzes the synthesis of NO, which aggravates neuronal injury [27]. Studies have found that treatment with geraniin reduces the increase in MDA content and significantly decreases SOD activity, thereby alleviating obesity and its pathophysiological sequelae by reducing oxidative stress [28]. However, no studies have shown that geraniin can reduce oxidative stress induced by cerebral I/R. In our study, we found that geraniin $(20 \mathrm{mg} / \mathrm{kg})$ significantly increased SOD activity, decreased LDH activity, and reduced MDA, NO, and nNOS contents in the sera and brain tissues of rats subjected to $\mathrm{MCAO} / \mathrm{R}$. The in vitro results were consistent with the in vivo findings. These results directly show that geraniin attenuates the cerebral I/R injury by suppressing oxidative stress.

Oxidative stress and apoptosis are involved in the pathological process of cerebral I/R injury. Inhibition of oxidative stress and apoptosis by drug intervention can benefit the treatment of cerebral I/R injury [29]. Accumulating evidence indicates that cerebral $\mathrm{I} / \mathrm{R}$ injury can cause the production and release of excessive free radicals and reactive oxygen species, which mediate the apoptosis pathway in neurons [30]. Neuronal apoptosis plays a key role in the cerebral I/ $\mathrm{R}$ injury [31]. Apoptosis is promoted by activation of the caspase family of cysteine proteases, which is one mechanism of programmed cell death regulation [32]. During the process of cerebral I/R injury, apoptosis is most closely related to the antiapoptotic protein $\mathrm{Bcl}-2$ and the proapoptotic protein Bax, which are members of the Bcl-2 family of proteins. Additionally, whether apoptosis occurs is determined by the $\mathrm{Bcl}-2 / \mathrm{Bax}$ expression ratio, and a decrease in the $\mathrm{Bcl}-2 / \mathrm{Bax}$ ratio has been reported to be related to an increase in apoptosis [33]. Furthermore, activation of caspase-3 leads to the occurrence of apoptotic events [34]. Studies have revealed that geraniin exerts significant inhibitory effects on tumor growth and markedly promotes cancer cell apoptosis by increasing the expression of Bax, caspase-3, and caspase- 9 and decreasing the level of Bcl-2 [35]. However, no studies have demonstrated that geraniin can play a neuroprotective role by exerting antiapoptotic effects in cerebral I/R injury. In the present study, geraniin obviously reduced the number of TUNEL-positive cells in vivo and decreased the rate of apoptosis in vitro. Moreover, in vivo and in vitro experiments indicated that treatment with geraniin significantly increased the expression level of Bcl-2 and decreased the expression levels of cleaved caspase- 3 and Bax, thus increasing the $\mathrm{Bcl}-2 / \mathrm{Bax}$ ratio. The above results indicate that geraniin can play a neuroprotective role by affecting the expression of apoptosis-related proteins.

Nrf2 is one of the important transcription factors in the endogenous defense system. The Nrf2-mediated signaling pathway can alleviate cerebral I/R injury through antioxidative stress, antiapoptosis, anti-inflammation, and other ways; it has been used as a key target for the treatment of ischemic stroke [36]. Studies have shown that after the cerebral I/R injury, oxygen free radicals can trigger Nrf2 phosphorylation and nuclear translocation, initiate the transcription of the downstream target gene $\mathrm{HO}-1$, and induce the production of a variety of endogenous antioxidant enzymes, thereby 


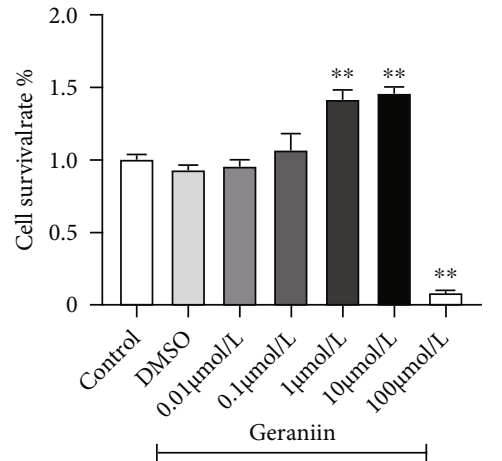

(a)

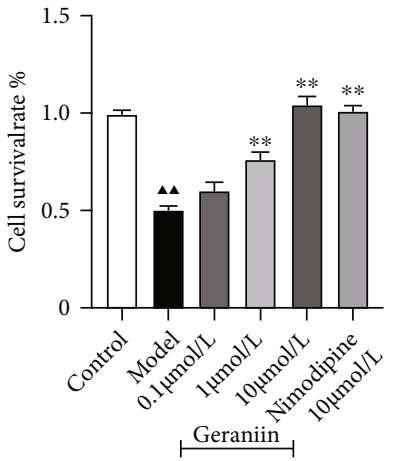

(b)

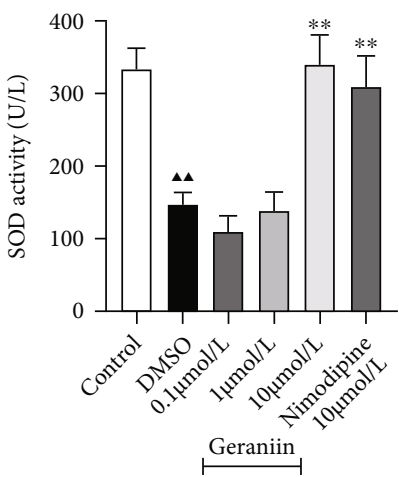

(c)

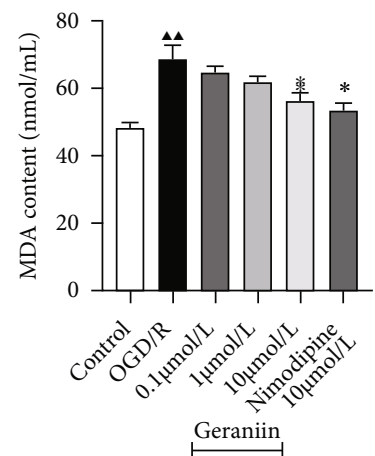

(e)

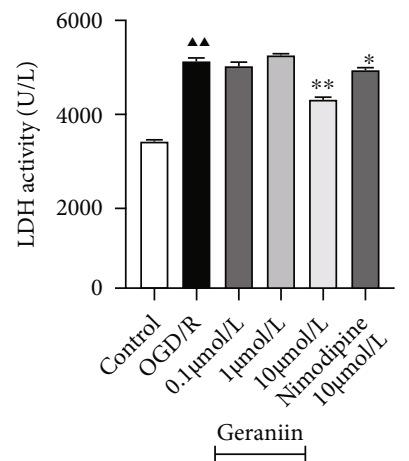

(d)

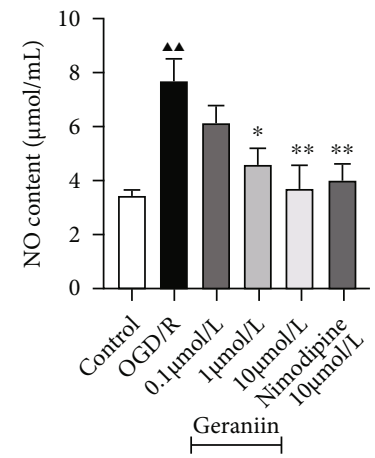

(f)

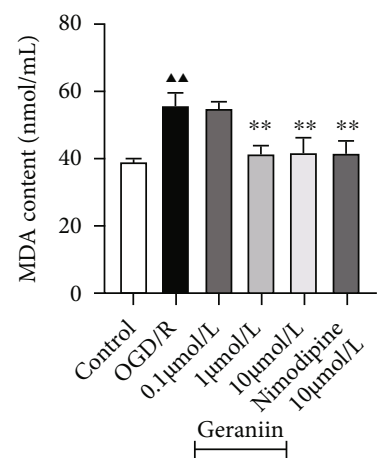

(g)

FIgURE 4: The effect of geraniin on cell viability and oxidative stress in PC12 cells after OGD/R. (a) The survival rate of geraniin-treated PC12 cells. (b) Effect of geraniin on the viability of PC12 cells subjected to OGD/R. (c-g) Effects of geraniin on SOD and LDH activity and MDA, NO, and nNOS contents in PC12 cells subjected to OGD/R. The data are expressed as the means \pm SEM $(n=8)$. ${ }^{\mathbf{\Delta}} P<0.01$ vs. the control group; ${ }^{*} P<0.05,{ }^{* *} P<0.01$ vs. the OGD/R group. 

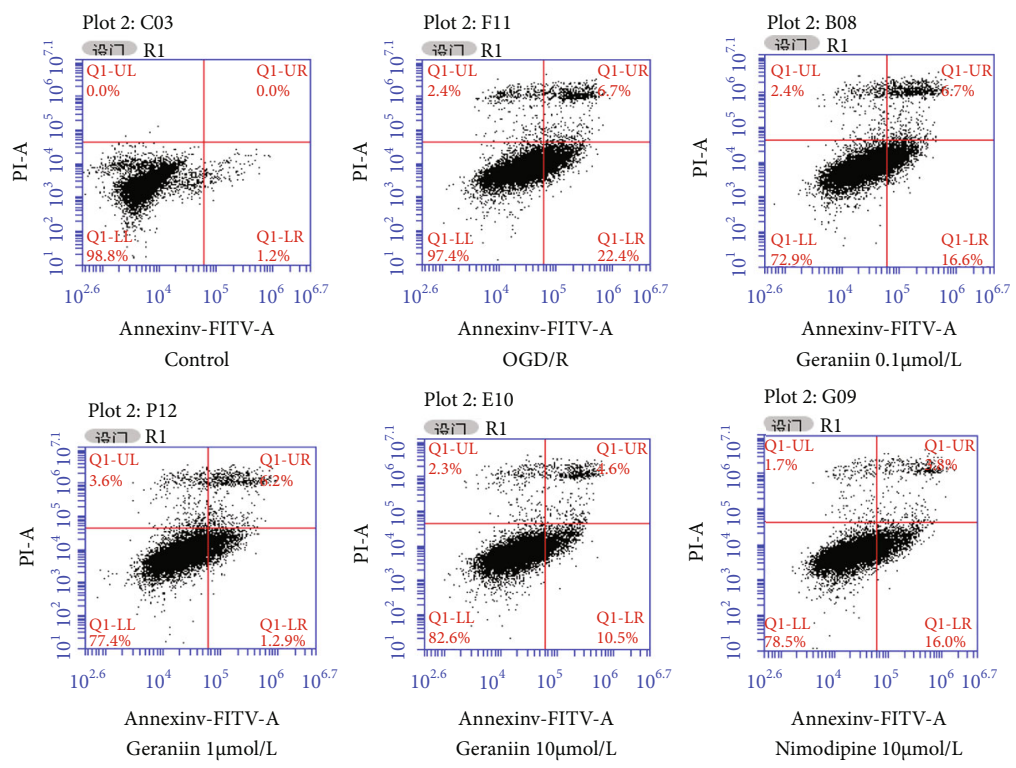

(a)

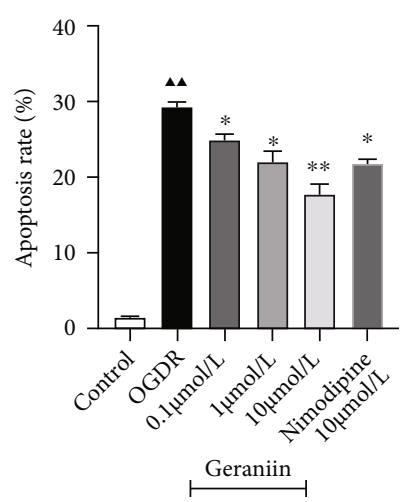

(b)

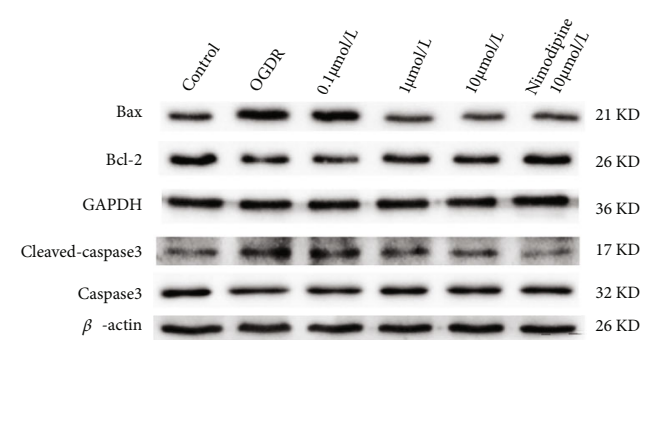

(c)

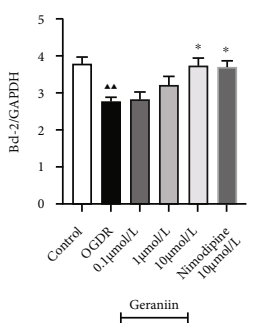

(e)

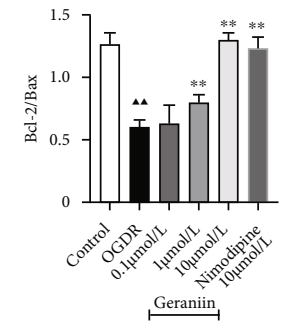

(f)

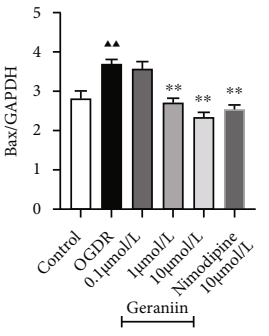

(d)

Figure 5: The effect of geraniin on OGD/R-induced apoptosis of PC12 cells. (a, b) Effect of geraniin on the OGD/R-induced cell apoptosis rate, as determined by flow cytometry. (c-f) The protein expression of Bax, Bcl-2, caspase-3, and cleaved caspase-3 was measured by Western blot analysis. The data are expressed as the means \pm SEM $(n=8)$. ${ }^{\boldsymbol{\Delta}} P<0.01$ vs. the control group; ${ }^{*} P<0.05,{ }^{* *} P<0.01$ vs. the OGD/R group.

reducing or eliminating the production of oxygen free radicals, resulting in redox balance in the body [37, 38]. Studies have found that in both the peri-infarct and core infarct regions, Nrf2 expression begins to increase at $2 \mathrm{~h}$, peaks at $8 \mathrm{~h}$, and then decreases at 24 and $72 \mathrm{~h}$ of reperfusion in a mouse transient middle cerebral artery (tMCAO) model [39]. These results suggest that activation of the Nrf2/HO1 pathway is associated with alleviating oxidative stressinduced damage. Meanwhile, the $\mathrm{Nrf} 2 / \mathrm{HO}-1$ signaling pathway is also involved in the regulation of apoptosis. Some studies confirmed that when the Nrf2 nuclear protein was inhibited in the $\mathrm{MCAO} / \mathrm{R}$ rat model, the expression of the proapoptotic protein Bax increased and the expression of the antiapoptotic protein $\mathrm{Bcl}-2$ decreased, thereby increasing the apoptosis rate $[40,41]$. Furthermore, inhibition of the Nrf2/HO-1 signaling pathway also can aggravate oxidative stress and apoptosis. Shih et al. [42] found that compared with wild-type mice, Nrf2-knockout mice treated with tMCAO exhibit decreased antioxidant enzyme activity, increased apoptosis, raised cerebral infarction area, and 


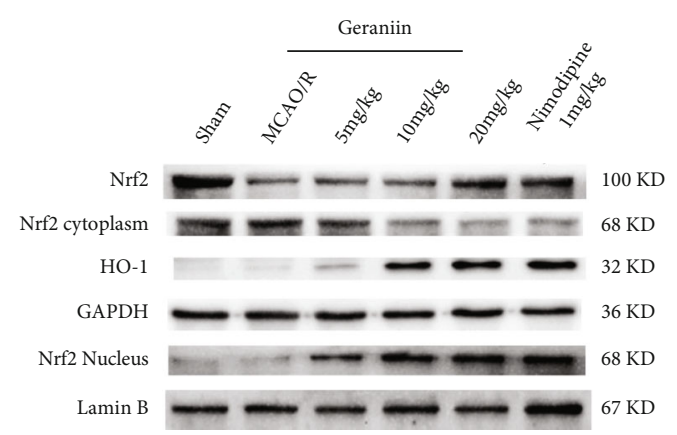

(a)

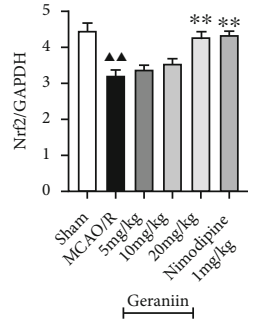

(b)

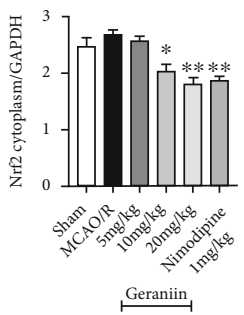

(c)

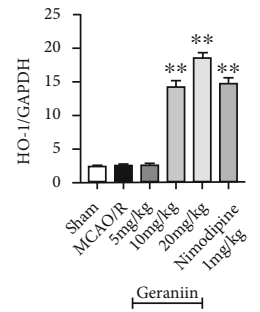

(d)

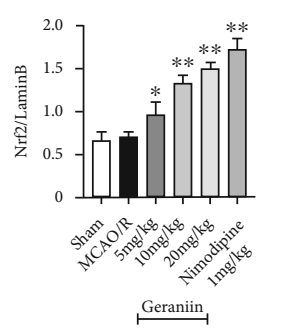

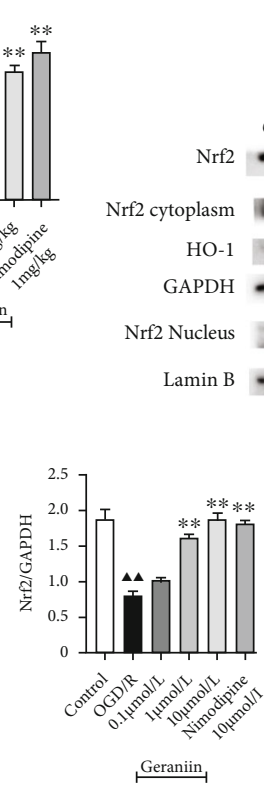

(g)

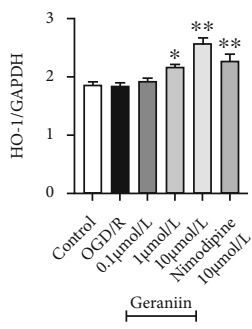

(i)

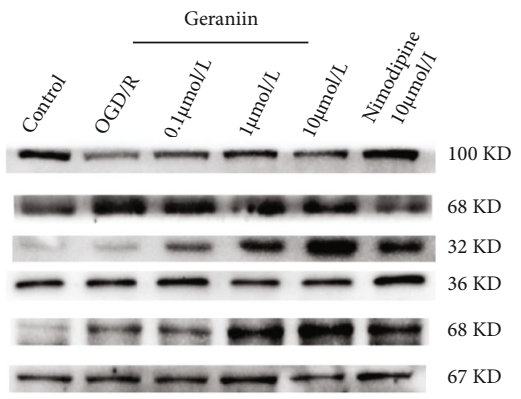

(f)

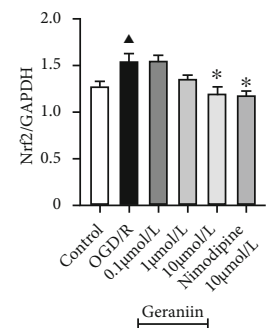

(h)

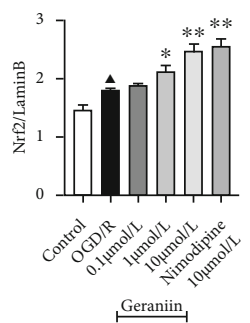

(j)

Figure 6: The effect of geraniin on the Nrf2/HO-1 signaling pathway in vivo and in vitro. (a-e) Protein expression levels of total Nrf2, cytoplasmic Nrf2, nuclear Nrf2, and HO-1 in the MCAO rat model after $72 \mathrm{~h}$ of reperfusion. (f-j) Protein expression levels of total Nrf2, cytoplasmic Nrf2, nuclear Nrf2, and HO-1 in PC12 cells exposed to OGD/R. The data are expressed as the means \pm SEM $(n=8)$. ${ }^{\wedge} P<0.01$ vs. the sham or control group; ${ }^{*} P<0.05,{ }^{* *} P<0.01$ vs. the MCAO/R or OGD/R group. 
aggravated behavioral deficits and cerebral I/R injury. Thus, the Nrf2/HO-1 signaling pathway plays an important role in antioxidant stress and apoptosis. In the present study, we found that geraniin significantly decreased the expression of Nrf2 in the cytoplasm in a concentration-dependent manner and increased total Nrf2, nuclear Nrf2, and HO-1 protein expression in vivo and in vitro. These results indicate that the neuroprotective mechanism of geraniin may be related to the activation of the $\mathrm{Nrf} 2 / \mathrm{HO}-1$ signaling pathway.

\section{Conclusion}

This study reports for the first time that geraniin exerts a neuroprotective effect against cerebral I/R injury both in vivo and in vitro, as indicated by improvements in neurological deficits, a reduction in the infarct volume, and decreases in oxidative stress and neuronal apoptosis, via regulation of the Nrf2/HO-1 signaling pathway. More experiments are needed in the future to reveal and elucidate the possible mechanisms underlying the neuroprotective effects of geraniin.

\section{Abbreviations \\ CIRI: Cerebral ischemia/reperfusion injury \\ MCAO/R: Middle cerebral artery occlusion/reperfusion \\ OGD/R: Oxygen glucose deprivation/reoxygenation \\ TTC: $\quad$ 2,3,5-Triphenyltetrazolium chloride \\ HE: Hematoxylin and eosin \\ SOD: $\quad$ Superoxide dismutase \\ LDH: $\quad$ Lactate dehydrogenase \\ MDA: Malondialdehyde \\ NO: $\quad$ Nitric oxide \\ nNOS: Neuronal nitric oxide synthase \\ Nrf2: $\quad$ Nuclear factor E2-related factor 2 \\ HO-1: Heme oxygenase-1.}

\section{Data Availability}

The data used to support the findings are available from the corresponding author upon request.

\section{Conflicts of Interest}

No conflicts of interests are declared by the authors.

\section{Authors' Contributions}

Yuan Yang and Bo He performed experiments and wrote the paper. Renhua Yang and Xiaochao Zhang analyzed the data. Xin Xia, Lu Chen, and Rui Li performed the animal experiments. Zhiqiang Shen revised article critically for important intellectual content. Peng Chen guided the experiment. Yuan Yang and Bo He contributed equally to this work.

\section{Acknowledgments}

This work was supported by the National Natural Science Foundation of China (Nos. 81660613 and 81860665), the
Joint Fund for the Department of Science and Technology of Yunnan Province-Kunming Medical University (Nos. 202001AY070001-157 and 2019FE001-191), the TenThousand Talents Program of Yunnan Province (No. YNWR-QNBJ-2019-137), and the Scientific Research Fund Project of Yunnan Education Department (2021Y325).

\section{References}

[1] J. D. Pandian, S. L. Gall, M. P. Kate et al., "Prevention of stroke: a global perspective," Lancet, vol. 392, pp. 1269-1278, 2018.

[2] D. Barthels and H. Das, "Current advances in ischemic stroke research and therapies," Biochimica et Biophysica ActaMolecular Basis of Disease, vol. 1866, no. 4, article 165260, 2020.

[3] M. Y. Wu, G. T. Yiang, W. T. Liao et al., "Current mechanistic concepts in ischemia and reperfusion injury," Cellular Physiology and Biochemistry, vol. 46, no. 4, pp. 1650-1667, 2018.

[4] F. Z. Caprio and F. A. Sorond, "Cerebrovascular disease: primary and secondary stroke prevention," The Medical Clinics of North America, vol. 103, no. 2, pp. 295-308, 2019.

[5] S. Liao, N. Apaijai, N. Chattipakorn, and S. C. Chattipakorn, "The possible roles of necroptosis during cerebral ischemia and ischemia / reperfusion injury," Archives of Biochemistry and Biophysics, vol. 695, article 108629, 2020.

[6] X. Meng, W. Xie, Q. Xu et al., "Neuroprotective effects of Radix Scrophulariae on cerebral ischemia and reperfusion injury via MAPK pathways," Molecules, vol. 23, no. 9, p. 2401, 2018.

[7] M. Valko, D. Leibfritz, J. Moncol, M. T. Cronin, M. Mazur, and J. Telser, "Free radicals and antioxidants in normal physiological functions and human disease," The International Journal of Biochemistry \& Cell Biology, vol. 39, no. 1, pp. 44-84, 2007.

[8] M. S. Sun, H. Jin, X. Sun et al., "Free radical damage in ischemia-reperfusion injury: an obstacle in acute ischemic stroke after revascularization therapy," Oxidative Medicine and Cellular Longevity, vol. 2018, no. 2, Article ID 3804979, p. 17, 2018.

[9] X. Sun and X. Cui, "Isorhapontigenin alleviates cerebral ische$\mathrm{mia} /$ reperfusion injuries in rats and modulated the PI3K/Akt signaling pathway," Naunyn-Schmiedeberg's Archives of Pharmacology, vol. 393, no. 9, pp. 1753-1760, 2020.

[10] Z. Huang, L. Guo, L. Huang, Y. Shi, J. Liang, and L. Zhao, "Baicalin-loaded macrophage-derived exosomes ameliorate ischemic brain injury via the antioxidative pathway," Materials Science \& Engineering. C, Materials for Biological Applications, vol. 126, no. 2, pp. 112-123, 2021.

[11] K. Fu, M. Chen, H. Zheng, C. Li, F. Yang, and Q. Niu, "Pelargonidin ameliorates MCAO-induced cerebral ischemia/reperfusion injury in rats by the action on the $\mathrm{Nrf} 2 / \mathrm{HO}-1$ pathway," Translational Neuroscience, vol. 12, no. 1, pp. 020-031, 2021.

[12] D. Huang, L. Yin, X. Liu et al., "Geraniin protects bone marrow-derived mesenchymal stem cells against hydrogen peroxide-induced cellular oxidative stress in vitro," International Journal of Molecular Medicine, vol. 41, no. 2, pp. 739748, 2018.

[13] X. Liu, J. Li, X. Peng et al., "Geraniin inhibits LPS-induced THP-1 macrophages switching to M1 phenotype via SOCS1/ NF- $\kappa$ B pathway," Inflammation, vol. 39, no. 4, pp. 14211433, 2016.

[14] P. Chen, F. Li, B. He et al., "Effects of geraniin on platelet aggregation and interactions between platelets and neutrophils," 
Journal of Kunming Medical University, vol. 33, no. 5, pp. 4-10, 2012.

[15] Y. Lu, B. He, X. Zhang et al., "Osteoprotective effect of geraniin against ovariectomy-induced bone loss in rats," Bioorganic \& Medicinal Chemistry Letters, vol. 25, no. 3, pp. 673-679, 2015.

[16] B. He, P. Chen, J. Yang et al., "Neuroprotective effect of 20(R)ginsenoside $\mathrm{Rg}_{3}$ against transient focal cerebral ischemia in rats," Neuroscience Letters, vol. 526, no. 2, pp. 106-111, 2012.

[17] J. B. Bederson, L. H. Pitts, M. Tsuji, M. C. Nishimura, R. L. Davis, and H. Bartkowski, "Rat middle cerebral artery occlusion: evaluation of the model and development of a neurologic examination," Stroke, vol. 17, no. 3, pp. 472-476, 1986.

[18] P. Wang, T. Y. Xu, Y. F. Guan et al., "Nicotinamide phosphoribosyltransferase protects against ischemic stroke through SIRT1-dependent adenosine monophosphate-activated kinase pathway," Annals of Neurology, vol. 69, no. 2, pp. 360-374, 2011.

[19] W. Chumboatong, S. Khamchai, C. Tocharus, P. Govitrapong, and J. Tocharus, "Agomelatine protects against permanent cerebral ischaemia via the Nrf2-HO-1 pathway," European Journal of Pharmacology, vol. 874, article 173028, 2020.

[20] B. He, P. Chen, Y. Xie et al., "20(R)-Ginsenoside Rg3 protects SH-SY5Y cells against apoptosis induced by oxygen and glucose deprivation/reperfusion," Bioorganic \& Medicinal Chemistry Letters, vol. 27, no. 16, pp. 3867-3871, 2017.

[21] W. Liu, Y. Miao, L. Zhang, X. Xu, and Q. Luan, "MiR-211 protects cerebral ischemia/reperfusion injury by inhibiting cell apoptosis," Bioengineered, vol. 11, no. 1, pp. 189-200, 2020.

[22] M. Y. Lu, J. R. Wu, R. B. Liang et al., "Upregulation of miR219a-5p Decreases Cerebral Ischemia/Reperfusion Injury In Vitro by Targeting Pde4d," Journal of Stroke and Cerebrovascular Diseases, vol. 29, no. 6, article 104801, 2020.

[23] K. Youn and M. Jun, "Geraniin protects PC12 cells against A $\beta 25-35$-mediated neuronal damage: involvement of NF- $\kappa \mathrm{B}$ and MAPK signaling pathways," Journal of Medicinal Food, vol. 23, no. 9, pp. 928-937, 2020.

[24] D. Wang, X. Dong, B. Wang, Y. Liu, and S. Li, "Geraniin attenuates lipopolysaccharide-induced cognitive impairment in mice by inhibiting toll-like receptor 4 activation," Journal of Agricultural and Food Chemistry, vol. 67, no. 36, pp. 1007910088, 2019.

[25] S. Orellana-Urzúa, I. Rojas, L. Líbano, and R. Rodrigo, "Pathophysiology of ischemic stroke: role of oxidative stress," Current Pharmaceutical Design, vol. 26, no. 34, pp. 4246-4260, 2020.

[26] R. Rodrigo, R. Fernández-Gajardo, R. Gutiérrez et al., "Oxidative stress and pathophysiology of ischemic stroke: novel therapeutic opportunities," CNS \& Neurological Disorders Drug Targets, vol. 12, no. 5, pp. 698-714, 2013.

[27] A. Francis and R. Baynosa, "Ischaemia-reperfusion injury and hyperbaric oxygen pathways: a review of cellular mechanisms," Diving and Hyperbaric Medicine, vol. 47, no. 2, pp. 110-117, 2017.

[28] A. P. Y. S. Chung, S. Gurtu, S. Chakravarthi, M. Moorthy, and U. D. Palanisamy, "Geraniin protects high-fat diet-induced oxidative stress in Sprague Dawley rats," Frontiers in Nutrition, vol. 5, pp. 17-23, 2018.

[29] K. Zhang, M. Tu, W. Gao, X. Cai, F. Song, Z. Chen et al., "Hollow Prussian blue nanozymes drive neuroprotection against ischemic stroke via attenuating oxidative stress, counteracting inflammation, and suppressing cell apoptosis," Nano Letters, vol. 19, no. 5, pp. 2812-2823, 2019.
[30] M. T. Lin and M. F. Beal, "Mitochondrial dysfunction and oxidative stress in neurodegenerative diseases," Nature, vol. 443, no. 7113, pp. 787-795, 2006.

[31] D. Radak, N. Katsiki, I. Resanovic et al., "Apoptosis and acute brain ischemia in ischemic stroke," Current Vascular Pharmacology, vol. 15, no. 2, pp. 115-122, 2017.

[32] Q. Liu and Y. Zhang, "PRDX1 enhances cerebral ischemiareperfusion injury through activation of TLR4-regulated inflammation and apoptosis," Biochemical and Biophysical Research Communications, vol. 519, no. 3, pp. 453-461, 2019.

[33] A. B. Uzdensky, "Apoptosis regulation in the penumbra after ischemic stroke: expression of pro- and antiapoptotic proteins," Apoptosis, vol. 24, no. 9-10, pp. 687-702, 2019.

[34] I. F. Muhammad, Y. Borné, O. Melander et al., "FADD (Fasassociated protein with death domain), caspase-3, and caspase- 8 and incidence of ischemic stroke," Stroke, vol. 49, no. 9, pp. 2224-2226, 2018.

[35] L. A. Zhou, T. B. Liu, and H. N. Lü, "Geraniin inhibits proliferation and induces apoptosis through inhibition of phosphatidylinositol 3-kinase/Akt pathway in human colorectal cancer in vitro and in vivo," Anti-Cancer Drugs, vol. 31, no. 6 , pp. 575-582, 2020.

[36] F. Sivandzade, A. Bhalerao, and L. Cucullo, "Cerebrovascular and neurological disorders: protective role of NRF2," International Journal of Molecular Sciences, vol. 20, no. 14, pp. 34333456, 2019.

[37] S. Jiang, C. Deng, J. Lv et al., "Nrf2 weaves an elaborate network of neuroprotection against stroke," Molecular Neurobiology, vol. 54, no. 2, pp. 1440-1455, 2017.

[38] F. Zhou, M. Wang, J. Ju et al., "Schizandrin A protects against cerebral ischemia-reperfusion injury by suppressing inflammation and oxidative stress and regulating the AMPK/Nrf2 pathway regulation," American Journal of Translational Research, vol. 11, no. 1, pp. 199-209, 2019.

[39] N. Tanaka, Y. Ikeda, Y. Ohta et al., "Expression of Keap 1-Nrf2 system and antioxidative proteins in mouse brain after transient middle cerebral artery occlusion," Brain Research, vol. 1370, pp. 246-253, 2011.

[40] A. Das, B. Gopalakrishnan, O. H. Voss, A. I. Doseff, and F. A. Villamena, "Inhibition of ROS-induced apoptosis in endothelial cells by nitrone spin traps via induction of phase II enzymes and suppression of mitochondria-dependent proapoptotic signaling," Biochemical Pharmacology, vol. 84, no. 4, pp. 486-497, 2012.

[41] J. Hur, S. C. Pak, B. S. Koo, and S. Jeon, "Borneol alleviates oxidative stress via upregulation of Nrf2 and Bcl-2 in SH-SY5Y cells," Pharmaceutical Biology, vol. 51, no. 1, pp. 30-35, 2013.

[42] A. Y. Shih, P. Li, and T. H. Murphy, "A small-moleculeinducible Nrf2-mediated antioxidant response provides effective prophylaxis against cerebral ischemia in vivo," The Journal of Neuroscience, vol. 25, no. 44, pp. 10321-10335, 2005. 